\title{
Phase in Optical Image Processing
}

\author{
Thomas J. Naughton ${ }^{a, b}$ \\ ${ }^{a}$ Department of Computer Science, National University of Ireland Maynooth, Maynooth, County Kildare, Ireland \\ ${ }^{b}$ University of Oulu, Oulu Southern Institute, RFMedia Laboratory, Vierimaantie 5, 84100 Ylivieska, Finland
}

\begin{abstract}
The use of phase has a long standing history in optical image processing, with early milestones being in the field of pattern recognition, such as VanderLugt's practical construction technique for matched filters, and (implicitly) Goodman's joint Fourier transform correlator. In recent years, the flexibility afforded by phase-only spatial light modulators and digital holography, for example, has enabled many processing techniques based on the explicit encoding and decoding of phase. One application area concerns efficient numerical computations. Pushing phase measurement to its physical limits, designs employing the physical properties of phase have ranged from the sensible to the wonderful, in some cases making computationally easy problems easier to solve and in other cases addressing mathematics' most challenging computationally hard problems. Another application area is optical image encryption, in which, typically, a phase mask modulates the fractional Fourier transformed coefficients of a perturbed input image, and the phase of the inverse transform is then sensed as the encrypted image. The inherent linearity that makes the system so elegant mitigates against its use as an effective encryption technique, but we show how a combination of optical and digital techniques can restore confidence in that security. We conclude with the concept of digital hologram image processing, and applications of same that are uniquely suited to optical implementation, where the processing, recognition, or encryption step operates on full field information, such as that emanating from a coherently illuminated real-world three-dimensional object.
\end{abstract}

Keywords: Optical image processing, optical processing, optical modulators, digital holography

PACS: 42.30.-d, 42.79.Hp, 42.79.Ta, 42.40.-I

\section{INTRODUCTION}

It has long been appreciated that spatial optical signals are the most natural means of representing continuous tone two-dimensional (2D) signals. There are many positive aspects to processing information using these (sometimes unwieldy and always inaccurate) physical signals instead of the more accurate digital electronic representations of 2D signals. These include the ability to concurrently modify all parts of an image (spatial light modulation), the capability to substitute space computational complexity for time computational complexity when performing certain transformations [1-5] (such as constant-time Fourier transformation with coherent light), the potential significant energy savings [6] (in both creating the signal and effecting the computation), and the ease with which analog signals can be digitised or resampled at an arbitrary frequency for subsequent digital electronic handling. The most common applications of optical image processing are pattern recognition and numerical matrix computations.

The use of phase is widespread in optical image processing, and is usually correlated with the use of coherent illumination in one's architecture. Invariably, a 2D image is encoded in the phase of an optical wavefront and decoded back again after processing using a phase contrast technique such as schlieren imaging or digital holography. The reasons for this can be because the spatial light modulators (SLMs) fundamentally work that way (e.g. LCD, LCoS, acousto-optic, and so on), because a complex-valued representation is necessary for the computation e.g. convolution using optical Fourier transformation, or because phase encoding offers advantages in terms of computational efficiencies or light power efficiencies.

Typically, those architectures that employ phase would be regarded as analog optical processing architectures, because each scalar is encoded in a single (quantised) phase value rather than encoded in a strictly digital representation where each scalar is represented by several base- $N$ digits. Digital optical computing paradigms typically specify the encoding of values in intensities, do not use coherent illumination, and do not rely on interference for computation, and as in electronics they encode in each time instance a scalar in each optical signal 
rather than an image. Although phase-encoded inputs can be processed in a digital framework [7], in this paper we find ourselves concentrating on analog optical processing systems that employ coherent illumination. The paper is structured as follows. We first review the use of phase in optical pattern recognition and then in analog optical numerical computation. We review an example of an optical image processing technique (optical encryption) that requires a complex-valued wavefront representation and sensing. Finally, we conclude with thoughts on the concept of three-dimensional image processing using digital holography.

\section{OPTICAL PATTERN RECOGNITION}

It could be argued that the field of optical information processing began in earnest with the realisation that spatially coherent laser light could be used to conveniently Fourier transform an image, allow one to modify the complex-valued spatial frequency components, and then inverse Fourier transform back to the spatial domain. This concept is called spatial (frequency) filtering [8-14], it is a generalisation that encompasses convolution and correlation operations, and it could be performed over 2D images in constant time while limited in speed only by the refresh rates of the input and output devices.

It first found application in the 1950s for parallel processing and analysis of the huge amounts of radar data produced at the time. The initial special-purpose spatial filtering systems performed optical Fourier transforms, performed image processing (for example, noise reduction and edge enhancement), and recognised patterns through correlation. The fundamentals of optical spatial filtering were formulated in that decade, and built upon previous work on optimum linear filtering in communication theory. Achieving the full potential of optical spatial filtering theory requires filters that are complex-valued, and a technique to fabricate such filters was first proposed by VanderLugt [11,15] and others [16], and made practical with alternative arrangements [17]. The techniques allow one to physically encode a complex-valued image on an amplitude-modulating SLM such as photographic film or an LCD panel.

Given a 2D spatial domain input image $a(x, y)$, which is Fourier transformed to give complex-valued image $A$ defined over spatial frequencies $(\alpha, \beta)$, and given 2D (usually complex) image $H$, the pointwise product of the two images,

$$
F[g(x, y)]=A(\alpha, \beta) H(\alpha, \beta),
$$

represents simultaneously the spatial frequency filtering of $a$ with filter $H$, the convolution of $a$ with convolution kernel $F^{-1}[H(-\alpha,-\beta)]$, and the correlation of $a$ with image $F^{-1}\left[H^{*}(\alpha, \beta)\right]$, where $F$ and $F^{-1}$ denote Fourier transformation and inverse Fourier transformation, respectively, and * denotes complex conjugate. The image $F[g(x, y)]$ would be inverse Fourier transformed to reveal a suitably correlated, convolved, or spatial frequency filtered output image. A significant proportion of analog optics' role in the area of computation through filtering concerns convolution and those signal processing operations derived from it. Convolution filters are used extensively in the digital signal processing world to perform such tasks as deblurring, restoration, enhancement, and recognition [18]. The possibility of performing constant time convolution operations using coherent light is a promising concept.

Research continued into this form of image-based computation. Many important image processing tasks were demonstrated at that time, from real-time tracking of moving objects [19,20], to telescope/microscope image deblurring [21,22]. Specificially in pattern recognition [23-30], effort focused on achieving systems invariant to scaling, rotation, out-of-plane rotation, deformation, and signal dependent noise, while retaining the existing invariance to translating, adding noise to, and obscuring parts of the input. Effort also went into injecting nonlinearities into these inherently linear systems to achieve wider functionality [31,32]. Improvements were made to the fundamental limitations of the VanderLugt filter, most notably the joint transform correlator architecture [33]. While one of the primary properties of the joint transform correlator is that it does not require the explicit phaseencoding of any signals, implicitly there is phase-encoding since the technique fundamentally relies on interference and an optical Fourier transform in its operation. Phase-only encoded versions of each architecture were demonstrated; advantages included higher light efficiencies and better discrimination capabilities (with multiple targets, cluttered scenes, or spatially-arranged filter banks the true target cross-correlation peaks can be difficult to locate in intensity-only input encodings).

A common example of an optical correlator's use in practical systems involved it being used as a front end to a generalised hybrid object recognition system. The optical processing component would quickly and efficiently identify regions of interest in a cluttered scene and pass these on to the slower but more accurate digital electronic 
components for false-alarm reduction, feature extraction, and classification. Today, the matched filter and the joint transform correlator are the two most widely used optical pattern recognition techniques [34-37].

At the same time as the development of sophisticated pattern recognition algorithms using coherent light, numerical computation techniques using values encoded in the complex amplitude were also developed, as explained next.

\section{ANALOG OPTICAL NUMERICAL COMPUTATION}

An important strand of image-based optical computation involved numerical calculations. The architectures are still image processing ones, but the interpretation one puts on the optical signals (that they represent vectors and matrices of numbers) mark them as being analog optical numberical computation. Digital optical numercical computation has also been well studied [38,39], but the archiectures are usually optical versions of electronic principles (for example, each optical channel at each timestep encodes a scalar rather than an image), and invariably do not utilise phase.

Matrix-vector and matrix-matrix multiplication systems were proposed and demonstrated [15,18]. The capability to expand a beam of light and to focus many beams of light to a common point directly corresponded to high fan out and fan in capabilities, respectively. Coherent light was used in many cases where complex-valued matrices were required and where the resolution of incoherent light proved unsatisfactory. Applications requiring matrix algebra benefited greatly from the tightly-coupled parallelism afforded by optics. An application that, further, was tolerant to the inherent inaccuracies and noise of analog optics was optical neural networks [40] including online neural learning in the presence of noise [41].

In most of these systems, phase was used implicitly in optical computations. By this we mean that there is noninterferometric intensity-based detection, but properties of the phase are necessary for the computation to take place. An example would be any computation that relies on an optical Fourier transform [4]. Explicit use of phase for basic arithmetic has been demonstrated; phase shifts can be introduced to a coherent wavefront by multiplying by a constant phase value and these could be used to effect numerical subtraction [7]. More wonderful techniques have been proposed for computationally intractable problems such as factoring and travelling salesman problem [42-45].

These fundamentally and/or practically are enabled through the utilisation of phase. Although they may complete these intractable problems in polynomial time, they usually require exponential amount of some other resource, such as space, wavelengths, or measurement accuracy. It is argued in [46] that an interesting way forward would be not to examine intractable problems and try to solve them efficiently in time (while using exponential amounts of some other resources such as pixels, or number of differentiable wavelengths), but to concentrate on applying resourcebounds to the computations and solve tractable problems super-efficiently, such as search problems [47].

Photons can also be used for quantum computation, and quantum computers using linear optical elements (such as mirrors, polarisers, beam splitters, and phase shifters) have been proposed and demonstrated [48-50] that (although routinely employ intensity-encoded input and outputs) fundamentally rely on interference.

\section{OPTICAL IMAGE ENCRYPTION}

Many techniques for the optical encryption of image data have been proposed and implemented in recent years [51-62]. Most perform encryption with a random phase mask positioned in the input, Fresnel, or Fraunhofer domain, or a combination of domains. These invariably produce a complex-valued encrypted image. Digital holography [6370] can be used to measure complex-valued wavefronts, and it has been applied to the encryption of 2D conventional (real-valued) images [57-59]. Of these, the techniques based on phase-shift interferometry [65,67,70] (PSI) make good use of detector resources in that they capture on-axis encrypted digital holograms [58,59]. The PSI technique has also been extended to the encryption of 3-D objects [60,61].

Because they have the distinct advantage of sending 2D complex data in parallel and carrying out otherwise time costly operations at great speeds, optical processors have found growing importance in data encryption. In [52], an optical encryption scheme is proposed called “double random phase encoding” (DRPE), which involves multiplying by two random phases in the input plane and in the Fourier domain. The authors show that if these random phases are statistically independent white noises, then the encrypted image is also a white noise. The random phase key located in the Fourier plane serves as the only key in this encryption scheme. The properties of this system and systems like it have been investigated extensively [71-75]. Various other linear optical systems have also been proposed in similar encryption architectures [76-84]. For example, the random phase keys can be located in a fractional Fourier domain [76-81] or a Fresnel domain [61,82,83]. The most general form of the linear canonical 
transform, implemented with any arbitrary quadratic phase system, has also been used in an encryption system that uses random phase as a key [84].

The DRPE method has been shown to have application in holographic data storage $[85,86]$. It has been successfully applied with angular multiplexing [87-90], and it has been observed that this methodology offers an improved performance over traditional angular multiplexing in terms of storage capacity [89.24] and angular selectivity [90]. This improvement is attributed to cross talk between adjacent images being reduced and has recently been both qualified and quantified using a Wigner-based approach [91].

In recent years there have been a number of proposed attacks [92-97] on DRPE-type encryption systems, including those systems that operate exclusively with phase [98,99]. Many of these attacks have shown that by only approximating the key one can get adequate decryption of encrypted images. In an effort to gain a deeper understanding of this system, and to overcome the vulnerability of DRPE systems to attack, it is necessary to investigate the parallels between this optical system and conventional cryptography [100-102]. All textbook conventional computer science encryption systems are vulnerable to attack. One way to counteract this is to use secure modes of operation into optical encryption.

The only study of its kind into secure modes of optical encryption [103] has considered DRPE, and considered all attacks proposed to date in one particular scenario. As is usual in cryptanalysis, only key security was considered; it is assumed that there is no security in the mechanism and that any potential attacker will know precisely how the key is used to effect encryption/decryption.

Modes were introduced in the following scenario. Consider a sequence of $m$ images that is to be optically encrypted, or equivalently, a stream of data that is very large compared to the input space of the DRPE apparatus. The output corresponding to such an input will be a sequence of encrypted images. The most secure way of encrypting these data is to use a separate encryption key for each image. However, using a separate key for each image is often impractical. In the scenario described here, the sender can transmit securely at most one or two phase masks to the receiver before sending the encrypted images over an insecure communication channel. The sender is therefore forced to reuse the same key for each image to be encrypted. However, this is vulnerable to attack. The modes of encryption presented in [103], each of increasing sophistication, allow the sender in this position some level of defence against the known attacks upon DRPE, as illustrated in Fig. 1.

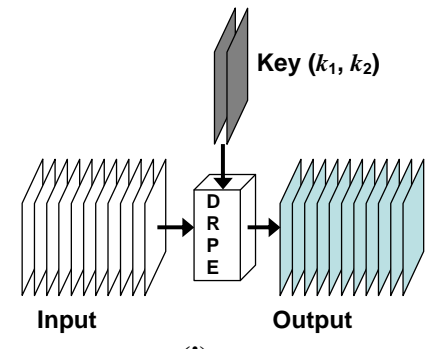

(i)

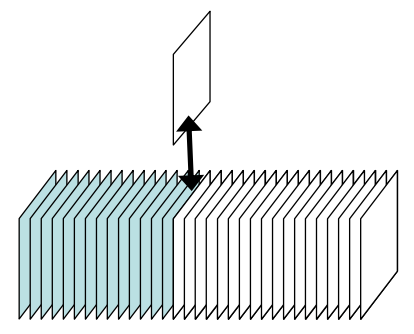

(iii)

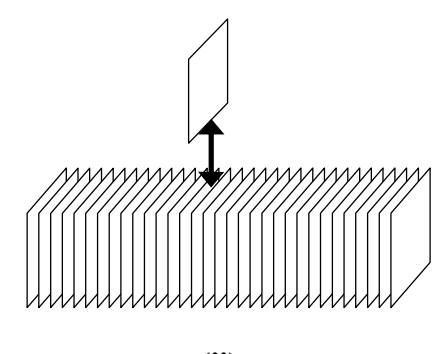

(ii)

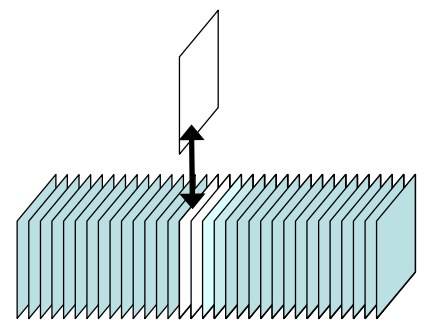

(iv)

FIGURE 1. Illustrations of DRPE with and without secure modes [103]: (i) A sequence of plaintext inputs (in white) is encrypted to ciphertext outputs (shaded). (ii) Without a suitably secure mode of encryption, if attackers obtain the key they can immediately decrypt the entire sequence forwards and backwards in time (previously encrypted and subsequently encrypted messages). (iii) In a secure mode, if attackers approximate the key with a single plaintext-ciphertext pair, only subsequent images

can be decrypted because elements of the mode calculation are not reversible. (iv) As introduced in [103], with a careful implementation of a secure mode, the propagation of errors from an attacker's approximation of the key will mean that only a very small number of subsequent images will be decrypted successfully, and the attacker will be forced to start the attack afresh on the subsequent images. 


\section{CONCLUSION}

A coherent wavefront is the most natural representation for a complex-valued image. Phase encodes directional information about a light field, and so 3D objects can be efficiently approximated with a 2D signal. Digital holography is one technique that records the full 2D complex field in a single camera frame instant, allowing it to be used for dynamic scenes. Because of holography's two stage process, a processed digital hologram can effect almost any imaging system. As such, a digital hologram can be considered a generalisation of a digital image, and digital hologram image processing can be considered as a generalisation of digital image processing.

Currently, in the field of hologram image processing it is most common to process the hologram reconstructions using conventional image processing techniques, to extract 3D information or interpret the 3D sensed scene. There are some notable exceptions where the hologram (complex-valued or otherwise) itself is processed directly [104108], however these exceptions are not common. A goal of digital hologram image processing must be to develop the understanding and tools to analyse the digital holograms directly. This could be done digitally for single-camera single-frame 3D image processing. The prevalence of high pixel-count SLMs, and the wealth of optical image processing knowledge built up over the past few decades, means that direct optoelectronic processing of 3D objects encoded in digital holograms could become economically viable. This could be a concrete realisation of the many advantages forseen for optical computing (for example, inherent computational parallelism, and energy efficiency) over those decades. The concept of digital hologram image processing does not preclude an optical processing implementation, where the strengths of each domain (electronic and optical) would be employed. Indeed, digital capture and optoelectronic reconstruction of holograms for the purpose of true 3D display is actively researched (see for example, http://www.digitalholography.eu). An online optoelectronic processor of this type would be capable of optical processing, recognition, or encryption step(s) operating on the full Fresnel field information, such as that directly emanating from a coherently illuminated real-world three-dimensional object.

\section{ACKNOWLEDGMENTS}

Some ideas in this manuscript have benefitted from discussions with Damien Woods. The research leading to these results has received funding from Science Foundation Ireland under the National Development Plan, and the European Community's Seventh Framework Programme FP7/2007-2013 under grant agreement no. 216105 (Real 3D).

\section{REFERENCES}

1. H. J. Caulfield. Proc. SPIE 1347, 566-572 (1990).

2. A. Louri and A. Post, Appl. Opt. 31, 5568-5583 (1992).

3. T. J. Naughton, Proc. SPIE 4109, 121-128 (2000).

4. J. H. Reif and A. Tyagi, Appl. Opt. 36, 7327-7340 (1997).

5. D. Woods, "Computational complexity of an optical model of computation", Ph.D. Thesis, National University of Ireland Maynooth, 2005.

6. H. J. Caulfield, OSA Optical Computing Technical Digest Series 9, 180-183 (1989).

7. D. G. Feitelson. Optical Computing: A survey for computer scientists, Cambridge, Massachusetts: MIT Press, 1988.

8. E. L. O’Neill, IRE Trans. Inf. Theory 2, 56-65 (1956).

9. G. L. Turin, IRE Trans. Inf. Theory 6, 311-329 (1960).

10. L. J. Cutrona, E. N. Leith, C. J. Palermo, and L. J. Porcello, IRE Trans. Inf. Theory 6, 386-400 (1960).

11. A. VanderLugt, IEEE Trans. Inform. Theory 10, 139-145 (1964).

12. L. J. Cutrona, E. N. Leith, L. J. Porcello, and W. E. Vivian, Proc. IEEE 54, 1026-1032 (1966).

13. A. VanderLugt, Proc. IEEE 62, 1300-1319 (1974).

14. E. N. Leith, Proc. IEEE 65, 18-28 (1977).

15. A. VanderLugt, Optical Signal Processing, New York: Wiley, 1992.

16. A. Kozma and D. L. Kelly, Appl. Opt. 4, 387 (1965).

17. J. W. Goodman, Introduction to Fourier Optics, Englewood, Colorado: Roberts \& Company, 2005, third edition.

18. M. A. Karim and A. A. S. Awwal, Optical Computing: An Introduction, New York: Wiley, 1992.

19. A. D. Gara, Appl. Opt. 18, 172-174 (1979).

20. J. Upatnieks, Appl. Opt. 22, 2798-2803 (1983).

21. G. W. Stroke, M. Halioua, F. Thon, and D. H. Willasch, Optik 41, 319-343 (1974).

22. G. Häusler, Optica Acta 24, 965 (1977).

23. D. P. Casasent and D. Psaltis, Opt. Eng. 15, 258-261 (1976). 
24. D. P. Casasent and D. Psaltis, Appl. Opt. 15, 1795-1799 (1976).

25. J. Caulfield and R. Haimes, Appl. Opt. 19, 181-183 (1980).

26. Y.-N. Hsu and H. H. Arsenault, Appl. Opt. 21, 4016-4019 (1982).

27. H. H. Arsenault, Y.-N. Hsu, and K. Chalasinska-Macukow, Opt. Eng. 23, 705-709 (1984).

28. D. P. Casasent, Appl. Opt. 23, 1620-1627 (1984).

29. B. Javidi and J. Wang, J. Opt. Soc. Am. A 12, 2604-2614 (1995).

30. S. Chang, H. H. Arsenault, P. Garcia-Martinez, and C. P. Grover, Appl. Opt. 39, 6641-6648 (2000).

31. B. Javidi, Appl. Opt. 28, 2358-2367 (1989).

32. B. Javidi, Appl. Opt. 29, 1215-1217 (1990).

33. C. S. Weaver and J. W. Goodman, Appl. Opt. 5, 1248-1249 (1966).

34. J. H. Feng, G. F. Chin, M. X. Wu, S. H. Yan, and Y. B. Yan. Opt. Lett. 20, 82-84 (1995).

35. J. L. Horner, Optical Signal Processing, San Diego:Academic Press, 1987.

36. X. J. Lu, F. T. S. Yu, and D. A. Gregory, Appl. Phys. B 51, 153-164 (1990).

37. F. T. S. Yu., Proc. SPIE 2969, 396-401 (1996).

38. A. Huang, Proc. IEEE 72, 780-786 (1984)

39. A. A. Sawchuk and T. C. Strand, Proc. IEEE 72, 758-779 (1984).

40. K.-Y. Hsu, H.-Y. Li, and D. Psaltis, Proc. IEEE 78, 1637-1645 (1990).

41. T. Naughton, Z. Javadpour, J. Keating, M. Klíma, and J. Rott, Opt. Eng. 38, 1170-1177 (1999).

42. K. Nitta, O. Matoba, and T. Yoshimura, Appl. Opt. 47, 611-616 (2008).

43. K. Nitta, N. Katsuta, and O. Matoba, LNCS 5882, 124-129 (2009).

44. M. Oltean, LNCS 4135, 217-227 (2006).

45. T. Haist and W. Osten, Opt. Express 15, 10473-10482 (2007). Erratum: Opt. Express 15, 12627-12627 (2007).

46. D. Woods and T. J. Naughton, Applied Mathematics and Computation 215, 1417-1430 (2009).

47. D. Woods, T. J. Naughton, LNCS 5172, 70-86 (2008).

48. N. J. Cerf, C. Adami, and P. G. Kwiat, Phys. Rev. A 57, R1477-R1480 (1998).

49. E. Knill, R. LaFlamme, and G. J. Milburn, Nature 409, 46-52 (2001).

50. T. B. Pittman, M. J. Fitch, B. C. Jacobs, and J. D. Franson, Phys. Rev. A, 68, 032316-3 (2003).

51. B. Javidi and J. L. Horner, Opt. Eng. 33, 1752-1756 (1994).

52. P. Réfrégier and B. Javidi, Opt. Lett. 20, 767-769 (1995).

53. L. G. Neto and Y. Sheng, Opt. Eng. 35, 2459-2463 (1996).

54. G. Unnikrishnan, J. Joseph, and K. Singh, Appl. Opt. 37, 8181-8186 (1998).

55. O. Matoba and B. Javidi, Opt. Lett. 24, $762-764$ (1999).

56. P. C. Mogensen and J. Glückstad, Opt. Lett. 25, 566-568 (2000).

57. B. Javidi and T. Nomura, Opt. Lett. 25, 28-30 (2000).

58. S. Lai and M. A. Neifeld, Opt. Commun. 178, 283-289 (2000).

59. E. Tajahuerce, O. Matoba, S. C. Verrall, and B. Javidi, Appl. Opt. 39, 2313-2320 (2000).

60. E. Tajahuerce and B. Javidi, Appl. Opt. 39, 6595-6601 (2000).

61. T.J. Naughton and B. Javidi, Opt. Eng. 43, 2233-2238 (2004).

62. N. K. Nishchal, J. Joseph, and K. Singh, Opt. Eng. 42, 1583-1588 (2003).

63. J. W. Goodman and R. W. Lawrence, Appl. Phys. Lett. 11, 77-79 (1967).

64. M. A. Kronod, N. S. Merzlyakov, and L. P. Yaroslavskii, Sov. Phys. Tech. Phys. 17, 333-334 (1972).

65. J. H. Bruning, D. R. Herriott, J. E. Gallagher, D. P. Rosenfeld, A. D. White, and D. J. Brangaccio, Appl. Opt. 13, 2693-2703 (1974).

66. T.-C. Poon and A. Korpel, Opt. Lett. 4, 317-319 (1979).

67. J. Schwider, B. Burow, K. E. Elsner, J. Grzanna, and R. Spolaczyk, Appl. Opt. 22, 3421-3432 (1983).

68. L. Onural and P. D. Scott, Opt. Eng. 26, 1124-1132 (1987).

69. U. Schnars and W. P. O. Ju“ptner, Appl. Opt. 33, 179-181 (1994).

70. I. Yamaguchi and T. Zhang, Opt. Lett. 22, 1268-1270 (1997).

71. B. Wang, C. C. Sun, W. C. Su, and A. E. T. Chiou, Appl. Opt. 39, 4788-4793 (2000).

72. F. Goudail, F. Bollaro, B. Javidi, and P. Refregier, J. Opt. Soc. Am. A 15, 2629-2638 (1998).

73. B. Javidi, N. Towghi, N. Maghzi, and S. C. Verrall, Appl. Opt. 39, 4117-4130 (2000).

74. D. S. Monaghan, U. Gopinathan, T. J. Naughton, and J. T. Sheridan, Appl. Opt. 46, 6641-6647 (2007).

75. D. S. Monaghan, G. Situ, U. Gopinathan, T. J. Naughton, and J. T. Sheridan, Appl. Opt. 47, 3808-3816 (2008).

76. G. Unnikrishnan and K. Singh, Opt. Eng. 39, 2853-2859 (2000).

77. G. Unnikrishnan, J. Joseph, and K. Singh, Opt. Lett. 25, 887-889 (2000).

78. S. Liu, L. Yu, and B. Zhu, Opt. Commun. 187, 57-63 (2001).

79. Y. Zhang, C. H. Zheng, and N. Tanno, Opt. Commun. 202, 277-285 (2002).

80. B. M. Hennelly and J. T. Sheridan, Optik 114, 251-265 (2003).

81. N. K. Nischal, G. Unnikrishnan, J. Joseph, and K. Singh, Opt. Eng. 42, 3566-3571 (2004).

82. O. Matoba and B. Javidi, Opt. Lett. 24, $762-764$ (1999).

83. G. Situ and J. Zhang, Opt. Commun. 232, 123-128 (2004).

84. G. Unnikrishnan and K. Singh, Opt. Commun. 193, 51-67 (2001). 
85. H. J. Caulfield, D. Psaltis, and G. Sincerbox, Holographic Data Storage, New York: Springer-Verlag, 2000.

86. L. Hesselink, S. S. Orlov, and M. C. Bashaw, Proc. IEEE 92, 1231-1280 (2004).

87. B. Javidi, G. Zhang, and J. Li, Appl. Opt. 36, 1054-1058 (1997).

88. O. Matoba and B. Javidi, Appl. Opt. 38, 7288-7293 (1999).

89. X. Tan, O. Matoba, T. Shimura, and K. Kuroda, Appl. Opt. 40, 4721-4727 (2001).

90. W. C. Su and C. H. Lin, Appl. Opt. 43, 2298-2304 (2004).

91. B. M. Hennelly, T. J. Naughton, J. B. McDonald, J. T. Sheridan, G. Unnikrishnan, D. P. Kelly, and B. Javidi, Opt. Lett. 32, 1060-1062 (2007).

92. A. Carnicer, M. Montes-Usategui, S. Arcos, and I. Juvells, Opt. Lett. 30, 1644-1646 (2005).

93. Y. Frauel, A. Castro, T. J. Naughton, and B. Javidi, Opt. Express 15, 10253-10265 (2007).

94. X. Peng, H. Wei, and P. Zhang, Opt. Lett. 31, 3261-3263 (2006).

95. U. Gopinathan, D. S. Monaghan, T. J. Naughton, and J. T. Sheridan, Opt. Express 14, 3181-3186 (2006).

96. X. Peng, P. Zhang, H. Wei, and B. Yu, Opt. Lett. 31, 1044-1046 (2006).

97. H.-Y. Lee, J.-P. Liu, C.-C. Chang, H.-F. Yau, and T.-C. Chang, Proc. SPIE 5560, 117-123 (2004).

98. N. Towghi, B. Javidi, and Z. Luo, J. Opt. Soc. Am. A 16, 1915-1927 (1999).

99. P. C. Mogensen and J. Glückstad, Opt. Lett. 25, 566-568 (2000).

100. W. Mao, Modern Cryptography, New York: Prentice Hall, 2004.

101. W. Stallings, Cryptography and Network Security, New York: Prentice Hall, 2005, 4th edition.

102. B. Schneier, Applied Cryptography, New York: Wiley, 1996, 2nd edition.

103. T. J. Naughton, B. M. Hennelly, and T. Dowling, J. Opt. Soc. Am. A 25, 2608-2617 (2008).

104. L. Onural and M. T. Özgen, J. Opt. Soc. Am. A 9, 252-260 (1992).

105. L. Denis, C. Fournier, T. Fournel, C. Ducottet, and D. Jeulin, Appl. Opt. 45, $944-952$ (2006).

106. S. Coëtmellec, D. Lebrun, and C. Özkul, Appl. Opt. 41, 312-319 (2002).

107. M. T. Özgen, Opt. Eng. 45, 035801 (2006).

108. S.-H. Lee, Y. Roichman, G.-R. Yi, S.-H. Kim, S.-M. Yang, A. van Blaaderen, P. van Oostrum, D. G. Grier, Opt. Express 15, 18275-18282 (2007). 